\title{
Scattering Formalism for Non-Localizable Fields
}

\author{
O. STEINMANN \\ SIN, Zürich
}

Received February 25, 1970

\begin{abstract}
We consider the theory of a non-localizable relativistic quantum field. Nonlocalizability means that the field is not a tempered distribution, but increases strongly for large momenta. Local commutativity can then not be satisfied. Instead we assume the existence of Green's functions with the usual analyticity properties. We show that in such a theory the $S$-matrix can be defined, and its elements can be expressed in terms of the fields by the usual reduction formulae.
\end{abstract}

\section{Introduction}

It is well known that the fields of relativistic quantum field theory $[1,2]$ are not functions of the space-time variable $x$ but must be considered as distributions, i.e. as linear functionals over a suitable space of test functions. On the exact nature of the acceptable test functions we have little or no physical information. For mathematical convenience it is usually assumed that the fields are tempered distributions. This assumption may, however, be too restrictive. In particular, there exist indications that the so-called non-renormalizable theories do not fit into this frame-work. Jaffe [3] showed that most of the theorems of axiomatic field theory can be derived for a more general class of fields, with a nontempered behaviour for large momenta. The allowed increase for large $p$, is, however, still not arbitrarily fast but is restricted by the condition that the field must be defined in $x$-space on test functions with compact support. Theories of this type are called "strictly localizable". Strict localizability is necessary if one wants the fields to be locally commuting.

The axiom of local commutativity has, however, no direct physical justification since the usually cited connection with causality is tenuous at best. Close inspection shows that the physically relevant form of causality (macrocausality), at least in its more acceptable formulations, depends mainly on the asymptotic behaviour of the Wightman functions in $x$-space, and practically not at all on their local properties. Translated into $p$-space this means that the smoothness properties for finite $p$ are important, while the increase for large $p$ is not. This increase is, then, of little significance. It manifests itself only in a restriction of the allowed test functions. 
It is therefore reasonable to consider more general cases than the ones treated by Jaffe, e.g. fields which are, in $p$-space, only defined on test functions with compact support. Such theories have been introduced in 1958 by Güttinger [4] but have come to the general attention only recently in connection with the work by Efimov and others on nonpolynomial Lagrangians ${ }^{1}$. Efimov constructs, starting from such a Lagrangian, a perturbative expansion of the Green's functions, which satisfies (hopefully) in every order the usual analyticity and unitarity requirements. In certain cases the increase of these functions in $p$-space turns out to be stronger than admitted by Jaffe. Nevertheless they define a field theory via the GLZ theorem, whose validity (with some obvious alterations in the formulation) in the present generalized case can easily be ascertained. (For the exact formulation and a proof of this theorem in the traditional tempered case we refer to an earlier paper by the present author [6].) It becomes then important to know whether the reverse of this theorem is true, i.e. whether something like the LSZ scattering formalism can be established. Can the $S$-matrix be defined, and if yes, can its matrix elements be expressed in the usual way in terms of the given Green's functions?

We shall show that the answer to these questions is yes. Assuming a certain regularity condition (to be formulated later) in $p$-space, we shall prove the cluster property, asymptotic conditions, and reduction formulae for the $S$-matrix.

As usual we consider the case of a single scalar hermitian field $A(x)$. This field shall satisfy the Wightman axioms with a particle spectrum (mass $m>0$ ) as formulated in Ref. [6], Postulates 1)-4), with the following exceptions. The distribution $A(x)$ is only defined on the space $Z$ [7], i.e. on test functions whose Fourier transforms have compact support ${ }^{2}$. The locality assumption (Postulate 4.d of [6]) can then no longer be formulated. It is replaced by a regularity condition in $p$-space, which we absorb in a new formulation of Postulate 6) stating the existence of retarded products. This postulate becomes now essential. We demand the existence of generalized retarded products (g.r.p.) as defined in [6], i.e. operator valued invariant distributions $G_{\mu}\left(x_{1}, \ldots, x_{n}\right)$ satisfying certain algebraic relations and symmetry properties. The support conditions (Postulate 6.vi) are replaced by a regularity property, which we shall now formulate.

1 The papers on this subject are too numerous to be quoted here. A full list of references can be traced from the report on the Trieste meeting on renormalization theory of August 1969 [5], in particular the contributions by F. Constantinescu, G. F. Efimov, H. Lehmann, A. Salam, B. Zumino.

${ }^{2}$ Our proofs can be easily extended to the test spaces lying between $Z$ and $\mathscr{S}$ that are used in the literature. 
Remember that $G_{\mu}$ is characterized by a function $\sigma_{\mu}(I)$ on the set of subsets $I$ of $\{2, \ldots, n\}$ with values in the sign set $\{+,-\}$. The ordinary retarded product $R$ is a special case of a g.r.p. belonging to the choice $\sigma(I)=-$ for all $I$.

Let $\Phi, \Psi$ be two vectors in the Hilbert space $\mathscr{H}$ of the theory, such that the matrix element $\left(\Phi, G_{\mu}\left(x_{1}, \ldots, x_{n}\right) \Psi\right)$ exists. The Fourier transform of this matrix element can be written as a distribution in the variables $p_{1}+\cdots+p_{n}=P, p_{2}, \ldots, p_{n}$. After integration with a test function in $P$ we obtain a distribution $\chi\left(p_{2}, \ldots, p_{n}\right)$. We demand:

Condition R. $\chi\left(p_{2}, \ldots, p_{n}\right)$ as defined above is analytic in the tube

$$
\mathscr{T}_{\mu}=\left\{\left(p_{2}, \ldots, p_{n}\right):\left(\sum_{i \in I} \operatorname{Im} p_{i}\right) \in V_{\sigma_{\mu}(I)}, \forall I\right\} .
$$

For each compact $K \subset R^{4(n-1)}$ and for all $\alpha, \beta$ with $1<\alpha<\infty, 0<\beta<\infty$ there exist a positive constant $c$ and non-negative integers $c_{i}$, such that

$$
\left|\chi\left(p_{i}\right)\right| \leqq c \prod_{i=2}^{n}\left|\operatorname{Im} p_{i 0}\right|^{-c_{i}}
$$

for $\left\{p_{i}\right\} \in \mathscr{T}_{\mu},\left\{\operatorname{Re} p_{i}\right\} \in K$, and $\alpha\left|\sum_{I} \operatorname{Im} p_{i}\right| \leqq\left|\sum_{I} \operatorname{Im} p_{i 0}\right| \leqq \beta, \forall I$.

$p_{i 0}$ is the 0 -component of the 4-vector $p_{i}, \boldsymbol{p}_{i}$ its space part.

The first part of this condition means that the "retarded" product $G_{\mu}$ has the same $p$-space-analyticity as in the tempered case, even though it has no support properties in $x$-space. The restriction (2) limits the possible divergence of $\chi$ on approaching real points. It guarantees the existence of the boundary value of $\chi$ as a distribution $\in \mathscr{D}^{\prime}$.

Note that we make no assumptions on the behaviour of $\chi$ for large $p_{i}$, real or imaginary. Here lies the difference between the non-localizable and the localizable case. For our purposes it would even be sufficient to assume analyticity of $\chi$ in the intersection of $\mathscr{T}_{\mu}$ with a neighbourhood of the real points, but we are here not concerned with finding optimal conditions.

We do, of course, not propose to introduce Condition $R$ as a new axiom in place of locality. This condition is too complicated and too far removed from any direct physical interpretation to be acceptable as a basic postulate. Instead we consider $R$ as a property that a given model may or may not possess, and show that in the models possessing it a $S$-matrix can be introduced as usual. The reverse is not true, i.e. $R$ is sufficient but not necessary for the existence of a scattering formalism. In a theory of the GLZ type, i.e. a theory defined by a set of Green's functions satisfying analyticity and unitarity, condition $R$ is fulfilled by the $G_{\mu}$ defined through their Haag expansion (Eq. (81) of [6]), the $13^{*}$ 
expansion coefficients being suitably chosen boundary values of the given Green's functions.

The time-ordered product $T\left(x_{1}, \ldots, x_{n}\right)$ is defined as in [6] with the help of a recursive relation connecting $R$ and $T$. The vacuum expectation values of $G_{\mu}, R, T$, are called $g_{\mu}, r, \tau$, respectively. The truncated part $\tau^{T}$ of $\tau$ (or of any other vacuum expectation value) is defined as usual via a cluster expansion. $\tilde{g}_{\mu}, \tilde{r}, \ldots$ are the Fourier transforms of $g_{\mu}, r, \ldots$. They are distributions in the Schwartz space $\mathscr{D}^{\prime}$.

From condition $R$ we deduce in the standard way [8] the existence of the Green's functions $H\left(p_{2}, \ldots, p_{n}\right)$ of whom $\tilde{g}_{\mu}$ and $\tilde{\tau}^{T}$ are boundary values:

Property $P$. Let $S$ be the set

$$
\begin{aligned}
& S=\left\{\left(p_{2}, \ldots, p_{n}\right):\left(\sum_{I} \operatorname{Im} p_{i}\right)^{2}<0\right. \\
& \left.\quad \text { or }\left(\sum_{I} \operatorname{Im} p_{i}=0\right) \text { and }\left(\sum_{I} \operatorname{Re} p_{i}\right)^{2}<m^{2}, \text { for all } I\right\} .
\end{aligned}
$$

Then there exists a function $H\left(p_{2}, \ldots, p_{n}\right)$, analytic in $S$, such that

$$
\begin{aligned}
\tilde{g}_{\mu}^{\prime}\left(\operatorname{Re} p_{2}, \ldots, \operatorname{Re} p_{n}\right) & =\lim _{\substack{\operatorname{Im} p_{i} \rightarrow 0 \\
\left(p_{i}\right) \in \mathscr{T}_{\mu}}} H\left(p_{2}, \ldots, p_{n}\right), \\
\tilde{\tau}^{T^{\prime}}\left(\operatorname{Re} p_{2}, \ldots, \operatorname{Re} p_{n}\right) & =\lim _{\operatorname{Im} p_{i} \rightarrow 0} H\left(p_{2}, \ldots, p_{n}\right),
\end{aligned}
$$

where the limit in (5) has to be taken from the set $\left(\operatorname{Re} \sum_{I} p_{i}, \operatorname{Im} \sum_{I} p_{i}\right) \geqq 0$
for all $I$. The prime in $\tilde{g}_{\mu}^{\prime}, \tilde{\tau}^{T^{\prime}}$ means dropping of the $\delta$-factor from momentum conservation:

$$
\tilde{g}_{\mu}\left(p_{1}, \ldots, p_{n}\right)=\delta^{4}\left(p_{1}+\cdots+p_{n}\right) \tilde{g}_{\mu}^{\prime}\left(p_{2}, \ldots, p_{n}\right) .
$$

\section{The Cluster Property}

The truncated Wightman function $W^{T}\left(x_{1}, \ldots, x_{n}\right)$ is said to possess the cluster property if the expression

$$
\psi\left(\boldsymbol{a}_{2}, \ldots, \boldsymbol{a}_{n}\right)=\int d x_{1} \ldots d x_{n} W^{T}\left(x_{1}, \ldots, x_{n}\right) \varphi\left(x_{1}, x_{2}+\boldsymbol{a}_{2}, \ldots, x_{n}+\boldsymbol{a}_{n}\right)
$$

is a tempered test function for all $\varphi \in Z$. Here $x+\boldsymbol{a}=\left(x^{0}, \boldsymbol{x}+\boldsymbol{a}\right)$. The cluster property for $g_{\mu}$ and $\tau^{T}$ is defined analogously.

By transforming (7) into $p$-space we see that

$$
\tilde{\psi}\left(\boldsymbol{p}_{2}, \ldots, \boldsymbol{p}_{n}\right)=\int d p_{20} \ldots d p_{n 0} \tilde{W}^{T^{\prime}}\left(p_{2}, \ldots, p_{n}\right) \tilde{\varphi}\left(p_{2}, \ldots, p_{n}\right)
$$

has to lie in the space $\mathscr{D}$ of $C^{\infty}$-functions with compact support if $\tilde{\varphi} \in \mathscr{D}$. $\widetilde{W}^{T^{\prime}}$ is defined as in (6). 
In this section we shall show that $\tilde{g}_{\mu}^{\prime}, \tilde{\tau}^{T^{\prime}}, \tilde{W}^{T^{\prime}}$ possess the cluster property (8). In the proof we shall not use condition $R$ but only its weaker corollary, the property $P$.

We need the following lemma.

Lemma 1. Let $H\left(k_{2}, \ldots, k_{n}\right), k_{j}=p_{j}+i q_{j}$, be analytic in $\mathscr{T}_{\mu}$ and satisfy the inequalities (2). Let $0<R<\infty$, and $\mathscr{T}_{\mu}^{R}=\mathscr{T}_{\mu} \cap\left\{\left|\boldsymbol{q}_{j}\right|<R, \forall j\right\}$. Let $\boldsymbol{D}$ be any derivation of arbitrary order in the space components $\boldsymbol{k}_{j}$, and $K \subset R^{4(n-1)}$ a compact.

Then there exists a differential operator $D^{0}$ in the energy components $k_{j 0}$, and a function $F\left(k_{j}\right)$ which is analytic in $\mathscr{T}_{\mu}^{\boldsymbol{R}}$ and continuous in $q_{j}=0,\left\{p_{j}\right\} \in K$, such that

in $\mathscr{T}_{\mu}^{R}$

$$
D H\left(k_{j}\right)=D^{0} F\left(k_{j}\right)
$$

For the sake of simplicity we give the proof for the special case $\mathscr{T}_{\mu}=\mathscr{T}_{+}$, the forward tube. The general case can be treated in exactly the same way.

Let $\bar{k}_{n 0}=i \bar{q}_{n 0}, \bar{q}_{n 0}>R$, be chosen once and for all. The 4-vector $\left(\bar{k}_{n 0}, \boldsymbol{k}_{n}\right)$ is in $\mathscr{T}_{+}^{R}$ if $\left|\boldsymbol{q}_{n}\right|<R$. For $\left(k_{2}, \ldots, k_{n}\right) \in \mathscr{T}_{+}^{R}$ we define

$$
G\left(k_{j}\right)=\int_{\bar{k}_{n_{0}}}^{k_{n_{0}}} d \zeta H\left(k_{2}, \ldots, k_{n-1}, \zeta, \boldsymbol{k}_{n}\right)
$$

with an integration path lying in $\mathscr{T}_{+}^{R} . G$ is evidently analytic, and

$$
H=\frac{\partial G}{\partial k_{n 0}} .
$$

For $q_{j}$ satisfying the restrictions of condition (2) we obtain

$$
|G| \leqq \int d|\zeta||H| \leqq c \prod_{2}^{n-1}\left|q_{j 0}\right|^{-c_{j}} \int d|\zeta||\operatorname{Im} \zeta|^{-c_{n}}
$$

We can choose an integration path pointing first in a real direction, then in a purely imaginary direction. Then

$$
|G| \leqq c^{\prime} \prod_{2}^{n-1}\left|q_{j 0}\right|^{-c_{j}} \cdot\left|q_{n 0}\right|^{-c_{n}+1}
$$

Iteration of this procedure gives

$$
H\left(k_{j}\right)=D^{0} F\left(k_{j}\right),
$$

where $F$ is analytic in $\mathscr{T}_{+}^{R}$ and bounded when approaching real points, and $D^{0}$ is a differential operator in the $k_{j 0}$ of sufficiently high order. The same result can be derived for $D H$, the order of $D^{0}$ of course depending on $\boldsymbol{D}$. 
The lemma follows then because boundedness of $D F$ implies the continuity of $F$.

We can now prove the cluster property for $g_{\mu}$. For $\tilde{\varphi}\left(p_{2}, \ldots, p_{n}\right) \in \mathscr{D}$, $\boldsymbol{D}$ as in Lemma 1, we have

$$
\begin{aligned}
\boldsymbol{D} \tilde{\varphi}\left(\boldsymbol{p}_{2}, \ldots, \boldsymbol{p}_{n}\right) & =\int d p_{j 0} \boldsymbol{D}\left[\tilde{g}_{\mu}^{\prime}\left(p_{j}\right) \tilde{\varphi}\left(p_{j}\right)\right] \\
& =\sum_{\boldsymbol{D}_{\alpha} \boldsymbol{D}_{\beta}=\boldsymbol{D}} c_{\alpha} \int d p_{j 0} \boldsymbol{D}_{\alpha} \tilde{g}_{\mu}^{\prime}\left(p_{j}\right) \boldsymbol{D}_{\beta} \tilde{\varphi}\left(p_{j}\right) \\
& =\sum c_{\alpha} \int d p_{j 0} D_{\alpha}^{0} F_{\alpha}\left(p_{j}\right) \boldsymbol{D}_{\beta} \tilde{\varphi}\left(p_{j}\right) \\
& =\sum c_{\alpha} \int d p_{j 0} F_{\alpha}\left(p_{j}\right) \check{D}_{\alpha}^{0} \boldsymbol{D}_{\beta} \tilde{\varphi}\left(p_{j}\right) .
\end{aligned}
$$

In the third line we have used Lemma 1, and the fourth line is obtained through integration by parts. $F$ is continuous, hence the integrals in the last line exist and are continuous in $\boldsymbol{p}_{j}$. Because of $\tilde{\varphi} \in \mathscr{D}$ they are of compact support, and thus the cluster property is proved.

The $4(n-1)$ dimensional $p$-space can be covered by a finite number of open sets, in each of which $\tilde{\tau}^{T^{\prime}}$ coincides with a g.r.f. $\tilde{g}_{\mu}^{\prime}$ [8]. Hence we can write $\tilde{\varphi}$ as a sum $\tilde{\varphi}=\sum_{\mu} \tilde{\varphi}_{\mu}$, such that $\tilde{\tau}^{T^{\prime}}=\tilde{g}_{\mu}^{\prime}$ in supp $\tilde{\varphi}_{\mu}$. The cluster property of $\tilde{\tau}^{T^{\prime}}$ follows then immediately from the cluster property of $g_{\mu}^{\prime}$.

$\tilde{W}^{T^{\prime}}$ can be written as a linear combination of generalized retarded functions multiplied with bounded $C^{\infty}$-functions $[9,10]$, and therefore has the cluster property.

\section{The Asymptotic Conditions}

The traditional proofs of the Haag-Ruelle asymptotic condition all suffer from one of two drawbacks. Either they work with regularized fields $[11,12]$, and this leads to difficulties in the formulation of the reduction formulae, or they use representation theorems for tempered distributions $[2,13]$ which do not hold in our non-tempered case. We shall therefore give a new proof which avoids these difficulties. It has at the same time the advantage of being applicable to the case of nonfactorizing multi-particle wave functions. For ease of notation we shall nevertheless only consider the factorizable case. The generalization is straightforward.

We use the notations of [6], in particular the following. Let $G$ be the set

$$
G=\left\{p: 0 \leqq p^{2} \leqq 4 m^{2}, p_{0} \geqq 0\right\} .
$$

The space of test functions $\in \mathscr{D}$ with support in $G$ is called $\mathscr{G}$. 
To $\tilde{f} \in \mathscr{G}$ we associate the operator

$$
A_{f}(t)=\int d^{4} p e^{-i t p^{-}} \tilde{f^{*}}(p) \tilde{A}(p),
$$

where

$$
p^{ \pm}=p_{0} \pm \omega(\boldsymbol{p}), \quad \omega(\boldsymbol{p})=\sqrt{\boldsymbol{p}^{2}+m^{2}} .
$$

Let

$$
\Phi(t)=A_{f_{1}}^{*}(t) \ldots A_{f_{n}}^{*}(t)|0\rangle .
$$

The Haag-Ruelle asymptotic condition states that

with

$$
\lim _{t \rightarrow \pm \infty} \Phi(t)=\Phi_{\text {out, in }}
$$

$$
\Phi_{\mathrm{ex}}=A_{f_{1}}^{\mathrm{ex}^{*}} \ldots A_{f_{n}}^{\mathrm{ex}}|0\rangle,
$$

$A^{\text {ex }}$ being free fields. The relation (19) holds also in the more general case in which some of the $A_{f}$ occur as annihilation operators, i.e. without *. For the sake of simplicity we shall only consider the special case given in (19).

Our proof follows the general outlines of Ruelle's procedure. We show that

$$
|\dot{\Phi}(t)|^{2} \sim|t|^{-3}
$$

for $|t| \rightarrow \infty$. In order to do this we develop the Wightman functions occurring in $(\dot{\Phi}(t), \dot{\Phi}(t))$ in cluster sums. We obtain a sum of products of terms of the form

$$
\begin{aligned}
X(t)= & \int \prod_{1}^{k}\left\{d p_{j} e^{-i t p_{\bar{j}}}\right\} \prod_{1}^{l}\left\{d q_{h} e^{-i t q_{h}^{+}}\right\} \tilde{f}\left(p_{1}, \ldots, q_{l}\right) \\
& \times \tilde{W}^{T}\left(p_{1}, \ldots, q_{l}\right),
\end{aligned}
$$

where $\tilde{f} \in \mathscr{D}$ and $k, l \leqq n$. We have to find the asymptotic behaviour of this expression.

The $p_{1}$-integration can be carried out with the help of the $\delta$-factor in $\tilde{W}^{T}$ (if $k=0$ we do the same for $q_{1}$ ):

$$
X(t)=\int d^{3} \boldsymbol{p}_{2} \ldots d^{3} \boldsymbol{p}_{k} d^{3} \boldsymbol{q}_{1} \ldots d^{3} \boldsymbol{q}_{l} \operatorname{Exp}\left\{i t E\left(\boldsymbol{p}_{j}, \boldsymbol{q}_{h}\right)\right\} \psi\left(\boldsymbol{p}_{j}, \boldsymbol{q}_{h}\right)
$$

where

$$
\begin{aligned}
E\left(\boldsymbol{p}_{j}, \boldsymbol{q}_{h}\right)= & \omega\left(\sum_{2}^{k} \boldsymbol{p}_{j}+\sum_{1}^{l} \boldsymbol{q}_{h}\right)+\sum_{2}^{k} \omega\left(\boldsymbol{p}_{j}\right)-\sum_{1}^{l} \omega\left(\boldsymbol{q}_{h}\right), \\
\psi\left(\boldsymbol{p}_{j}, \boldsymbol{q}_{h}\right)= & \int d p_{20} \ldots d p_{k 0} d q_{10} \ldots d q_{l 0} \tilde{W}^{T^{\prime}}\left(p_{2}, \ldots, q_{l}\right) \\
& \times \tilde{f}\left(-\sum_{2}^{k} p_{j}-\sum_{1}^{l} q_{h}, p_{2}, \ldots, q_{l}\right) .
\end{aligned}
$$

According to the results of the preceding section $\psi$ is a function from $\mathscr{D}$. 
We need to know the manifold $\mathscr{M}$ on which $E$ is stationary. $\mathscr{M}$ is determined by the conditions

$$
\begin{aligned}
& \frac{\partial E}{\partial \boldsymbol{p}_{i}}=\frac{\sum \boldsymbol{p}_{j}+\sum \boldsymbol{q}_{h}}{\omega\left(\sum \boldsymbol{p}_{j}+\sum \boldsymbol{q}_{h}\right)}+\frac{\boldsymbol{p}_{i}}{\omega\left(\boldsymbol{p}_{i}\right)}=0, \\
& \frac{\partial E}{\partial \boldsymbol{q}_{g}}=\frac{\sum \boldsymbol{p}_{j}+\sum \boldsymbol{q}_{h}}{\omega\left(\sum \boldsymbol{p}_{j}+\sum \boldsymbol{q}_{h}\right)}-\frac{\boldsymbol{q}_{g}}{\omega\left(\boldsymbol{q}_{g}\right)}=0 .
\end{aligned}
$$

These conditions are equivalent to

$$
\sum_{2}^{k} \boldsymbol{p}_{j}+\sum_{1}^{l} \boldsymbol{q}_{h}=-\boldsymbol{p}_{i}=\boldsymbol{q}_{g}
$$

for $i=2, \ldots, k ; g=1, \ldots, l$. For $k \neq l$ ("regular case") (27) is only satisfied for $\boldsymbol{p}_{i}=\boldsymbol{q}_{g}=0$, i.e. $\mathscr{M}$ consists of a single point. For $k=l$ ("singular case") (27) is solved by $\boldsymbol{p}_{i}=-\boldsymbol{q}_{g}=\boldsymbol{p}, \boldsymbol{p}$ an arbitrary 3 -vector. In this case $\mathscr{M}$ is a 3-dimensional plane.

In the regular case we introduce suitable linear combinations $u_{1}, \ldots, u_{r}$ $(r=3(k+l-1))$ of the components of the $\boldsymbol{p}_{i}, \boldsymbol{q}_{g}$ as new variables. In the singular case we take the 3 components of

$$
\boldsymbol{p}=\frac{\sum \boldsymbol{p}_{j}-\sum \boldsymbol{q}_{h}}{k+l-1}
$$

and again suitable linear combinations $u_{1}, \ldots, u_{r}(r=3(k+l-2))$ of the $\boldsymbol{p}_{i}, \boldsymbol{q}_{g}$, which are chosen such that they parametrize the orthogonal complement of $\mathscr{M}$ and vanish on $\mathscr{M}$. The meaning of the word "suitable" will become apparent later.

In both cases we can develop $E$ into a power series in the $u_{i}$ :

$$
E(u)=E_{0}+E^{\prime}(u), \quad E^{\prime}(u)=E_{2}(u)+E_{h}(u) .
$$

Here $u$ stands for the $r$-tuplet $\left\{u_{i}\right\}$, and $E_{0}=(k-l) m . E_{2}$ is a non-degenerate quadratic form whose coefficients depend in the singular case on $p$, and $|u|^{2}=\sum u_{i}^{2}$.

$$
E_{h}(u)=O\left(|u|^{3}\right),
$$

In the singular case we define an expression $X(t, p)$ by integrating in (23) only over the $u_{i}$. The estimates which we are going to find will turn out to be uniform in $p$, and will not be invalidated by the final $p$-integration. We can therefore neglect the $p$-dependence of $E_{2}$ and $E_{h}$.

The unitary factor $e^{i t E_{0}}$ is irrelevant for our purposes and will be dropped. We have then to estimate expressions of the form

$$
F(t)=\int d^{r} u \psi(u) \operatorname{Exp}\left\{i t E^{\prime}(u)\right\} .
$$


$F$ can be written in the form

$$
\begin{aligned}
F(t) & =\int d \lambda e^{i t \lambda} G(\lambda), \\
G(\lambda) & =\int d^{r} u \delta\left(E^{\prime}(u)-\lambda\right) \psi(u) .
\end{aligned}
$$

$G$ has a compact support. The asymptotic behaviour of $F$ depends on the local singularities of $G$. These we shall now determine.

Define

$$
\left.\begin{array}{rl}
H_{\alpha}^{1}(x) & =\frac{(-1)^{\alpha+1}}{\alpha !} \theta(x) x^{\alpha} \\
H_{\alpha}^{2}(x) & =\frac{(-1)^{\alpha}}{\alpha !} \theta(-x) x^{\alpha}
\end{array}\right\} \quad \alpha \geqq 0,
$$

We shall use the notation $H_{\alpha}$ in relations which hold for both $H_{\alpha}^{1}$ and $H_{\alpha}^{2}$. All $H_{\alpha}$ occurring in an equation have to carry the same upper index.

$H_{\alpha}(x)$ is $(\alpha-1)$ times continuously differentiable. We have

$$
\begin{gathered}
\frac{\partial}{\partial \lambda} H_{\alpha}\left(E^{\prime}(u)-\lambda\right)=H_{\alpha-1}\left(E^{\prime}-\lambda\right), \\
\delta\left(E^{\prime}-\lambda\right)=\frac{\partial^{\alpha+1}}{\partial \lambda^{\alpha+1}} H_{\alpha}\left(E^{\prime}-\lambda\right)
\end{gathered}
$$

for all $\alpha \geqq 0$, and

$$
\frac{\partial}{\partial \lambda} H_{\alpha}\left(E^{\prime}(u)-\lambda\right)=-\left(\frac{\nabla E^{\prime}}{\left|\nabla E^{\prime}\right|^{2}}, \nabla H_{\alpha}\left(E^{\prime}-\lambda\right)\right) .
$$

Here $\nabla=\operatorname{grad}_{u}$, and $(\ldots, \ldots)$ is the scalar product in the $R^{r}$.

We define

$$
E(u)=\nabla E^{\prime}(u)=E_{1}(u)+E_{h}(u),
$$

$\boldsymbol{E}_{1}$ homogeneous of first degree, $\boldsymbol{E}_{h}=\boldsymbol{O}\left(|u|^{2}\right)$.

We substitute (36) into the definition (33), use (37) and integrate $(\alpha+1)$ times by part:

$$
\begin{aligned}
G(\lambda) & =\int d^{r} u H_{\alpha}\left(E^{\prime}-\lambda\right) \psi_{\alpha}(u), \\
\psi_{\alpha}(u) & =\left(\nabla, \frac{\boldsymbol{E}}{|\boldsymbol{E}|^{2}}\right)^{\alpha+1} \psi(u) .
\end{aligned}
$$

Here $(\nabla, \boldsymbol{F}) \psi=\psi \operatorname{div} \boldsymbol{F}+(\boldsymbol{F}, \operatorname{grad} \psi)$.

Assume $\lambda>0$. We use $H_{\alpha}^{1}$ in (39). $E^{\prime}$, and therefore $H_{\alpha}^{1}\left(E^{\prime}-\lambda\right)$ vanish in $u=0$. But $\psi_{\alpha}$ is $C^{\infty}$ outside the origin, hence $G(\lambda)$ exists. The same type of argument shows that any arbitrarily high derivative of $G$ exists. We 
have only to choose $\alpha$ large enough and use (35). Substitution of $H_{\alpha}^{2}$ into (39) yields the same result for $\lambda<0$. Thus we obtain a first result: $G(\lambda)$ is infinitely differentiable everywhere except in $\lambda=0$.

In the origin $\psi_{\alpha}$ has a singularity of order $2 \alpha+2$. We can expand $\psi_{\alpha}$ as follows:

$$
\psi_{\alpha}(u)=\sum_{\mu=-2 \alpha-2}^{-1} \psi_{\alpha}^{\mu}(u)+\psi_{\alpha}^{R}(u),
$$

with $\psi_{\alpha}^{\mu}$ homogeneous of degree $\mu$ and $C^{\infty}$ outside $0, \psi_{\alpha}^{R}$ continuous. The first term in this expansion is

$$
\psi_{\alpha}^{-2 \alpha-2}(u)=\psi(0) \cdot\left(\nabla, \frac{\boldsymbol{E}_{2}}{\left|\boldsymbol{E}_{2}\right|^{2}}\right)^{\alpha+1}
$$

in evident notation. The integral (39) can be taken over a sphere of radius $\varrho<\infty$, which contains the support of $\psi$. We obtain for the contribution $G_{R}$ of $\psi_{\alpha}^{R}$ to this integral

$$
\frac{\partial^{\beta} G_{R}}{\partial \lambda^{\beta}}=\int_{|u| \leqq \varrho} d^{r} u H_{\alpha-\beta}\left(E^{\prime}-\lambda\right) \psi_{\alpha}^{R} .
$$

This exists and is continuous in $\lambda=0$ for $\beta \leqq \alpha$, i.e. $G_{R}$ is $\alpha$ times continuously differentiable. Hence $G_{R}$ can be made arbitrarily regular by choosing a sufficiently high $\alpha$. We shall therefore drop the $\psi_{\alpha}^{R}$-term.

$H_{\alpha}$ can be expanded as follows:

$$
H_{\alpha}\left(E^{\prime}-\lambda\right)=\sum_{v=0}^{K} H_{\alpha-v}\left(E_{2}-\lambda\right) \frac{\left(E_{h}\right)^{v}}{v !}+H_{\alpha}^{R}(\lambda, u),
$$

with $K \leqq \alpha-1$, and $H_{\alpha}^{R}(0, u)=O\left(|u|^{2 \alpha+K+1}\right)$. It is easy to see that

$$
\frac{\partial^{\beta} H_{\alpha}^{R}}{\partial \lambda^{\beta}}=H_{\alpha-\beta}^{R}=O\left(|u|^{2 \alpha-2 \beta+K+1}\right)
$$

for $\lambda=0$. The contribution of $H^{R}$ to $G$ is, then, $K+r$ times differentiable. It can be made arbitrarily regular through the choice of $K$ (and $\alpha$ ) and can therefore again be dropped.

Finally we develop the product $\psi_{\alpha}^{\mu}\left(E_{h}\right)^{v}$ into a series of homogeneous terms:

$$
\psi_{\alpha}^{\mu}\left(E_{h}\right)^{v}=\sum_{\sigma=\mu+3 v}^{M} \psi_{\alpha, \mu, v}^{\sigma}(u)+\psi_{\alpha, \mu, v}^{R}(u)
$$

with $\psi_{\alpha, \mu, v}^{\sigma}$ homogeneous of degree $\sigma, \psi_{\alpha \mu \nu}^{R}=O\left(|u|^{M}\right)$. The last term can again be dropped for sufficiently high $M$. 
The remaining contributions to $G$ are

$$
G_{\alpha, \mu, v, \sigma}(\lambda)=\int_{|u| \leqq \varrho} d^{r} u H_{\alpha-v}\left(E_{2}-\lambda\right) \psi_{\alpha, \mu, v}^{\sigma} .
$$

The most singular term comes from

as given by (42).

$$
\psi_{\alpha,-2 \alpha-2,0}^{-2 \alpha-2}(u)=\psi_{\alpha}^{-2 \alpha-2}(u)
$$

The $\beta^{\text {th }}$ derivative

$$
\frac{\partial^{\beta}}{\partial \lambda^{\beta}} G_{\alpha, \mu, v, \sigma}=\int d^{r} u H_{\alpha-\nu-\beta}\left(E_{2}-\lambda\right) \psi_{\alpha, \mu, v}^{\sigma}
$$

exists in $\lambda=0$ for $2 \beta<r+2 \alpha-2 v+\sigma$. Hence $G_{\alpha, \mu, v, \sigma}$ is

$$
\beta_{\alpha v \sigma}=\left[\frac{r+2 \alpha-2 v+\sigma}{2}\right]
$$

times differentiable, where $[x]$ is the largest integer with $[x]<x$. Because of $\sigma \geqq \mu+3 v$ and $\mu \geqq-2 \alpha-2$ we have

$$
\beta_{\alpha v \sigma} \geqq\left[\frac{r+v-2}{2}\right] \text {. }
$$

The smallest $\beta$ belongs to $v=0, \mu=-2 \alpha, \sigma=-2 \alpha$, and is $\left[\frac{r}{2}-1\right]$. Thus $G(\lambda)$ is $\left[\frac{r}{2}-1\right]$ times continuously differentiable.

The $\gamma^{\text {th }}$ derivative $\left(\gamma>\beta_{\alpha v \sigma}\right)$ of $G_{\alpha, \mu, v, \sigma}$ is

$$
G_{\alpha, \mu, v, \sigma}^{\gamma}=\int d^{r} u H_{\alpha-\nu-\gamma}\left(E_{2}-\lambda\right) \psi_{\alpha, \mu, v}^{\sigma} .
$$

For $\lambda>0$ we use $H^{1}$. The substitution $u=\sqrt{\lambda} v$ yields

$$
G_{\alpha, \mu, v, \sigma}^{\nu}=\sqrt{\lambda}^{r+2 \alpha-v-\gamma+\sigma} \int_{|v| \leqq \varrho / \sqrt{\lambda}} d^{r} v H_{\alpha-\nu-\gamma}^{1}\left(E_{2}(v)-1\right) \psi_{\alpha, \mu, v}^{\sigma}(v) .
$$

This integral exists for $\lambda>0$. The limit $\lambda \rightarrow 0$ exists if $r+2(\alpha-v-\gamma)$ $+\sigma<0$. In this case we can develop the integral into a power series in $\sqrt{\lambda}$ and obtain

$$
G_{\alpha, \mu, v, \sigma}^{\gamma}=\sqrt{\lambda}^{r+2 \alpha-\nu-\gamma+\sigma}\left\{\sum_{0}^{N} c_{x} \sqrt{\lambda}^{x}+O\left(\sqrt{\lambda}^{N+1}\right)\right\} .
$$

For $r+2(\alpha-v-\gamma)+\sigma=0$ we obtain

$$
G_{\alpha, \mu, v, \sigma}^{\gamma}=\sum_{0}^{N} c_{\varkappa} \sqrt{\lambda}^{\varkappa}+\log \lambda \sum_{0}^{N} d_{\varkappa} \sqrt{\lambda}^{\varkappa}+O\left(\sqrt{\lambda}^{N+1} \cdot \log \lambda\right)
$$


whence

$$
\begin{aligned}
G_{\alpha, \mu, v, \sigma}(\lambda)= & \lambda^{\beta_{\alpha \nu \sigma}}\left[\sum_{0}^{N} c_{\chi} \sqrt{\lambda}^{\varkappa}+\log \lambda \sum_{0}^{N} d_{\varkappa} \sqrt{\lambda}^{\chi}\right] \\
& +O\left(\sqrt{\lambda}^{2 \beta_{\alpha \nu \sigma}+N+1} \log \lambda\right) .
\end{aligned}
$$

A similar expansion, with $\lambda$ replaced by $|\lambda|$, holds for $\lambda<0$.

The most singular term is

$$
\begin{aligned}
G_{\alpha,-2 \alpha, 0,-2 \alpha}(\lambda) & =\psi(0) \int d^{r} u H_{\alpha}\left(E_{2}-\lambda\right)\left(\nabla, \frac{\boldsymbol{E}_{2}}{\left|\boldsymbol{E}_{2}\right|^{2}}\right)^{\alpha+1} \\
& =\psi(0) \int_{|u| \leqq \varrho} d^{r} u \delta\left(E_{2}-\lambda\right)+\text { boundary terms . }
\end{aligned}
$$

The last equality is obtained by reversing the operations which lead from (33) to (39). The boundary terms from the partial integrations are infinitely differentiable in $\lambda=0$ and are therefore irrelevant.

If $E_{2}$ is positive definit, i.e. $E_{2}=\sum u_{i}^{2}=u^{2}$ for suitably chosen parameters $u_{i}$, then

$$
\int_{|u| \leqq R} d^{r} u \delta\left(E_{2}-\lambda\right)=\text { const. } \theta(\lambda) \lambda^{(r-2) / 2}
$$

for $\lambda<R$. There is no logarithmic term. An analogous calculation for indefinite $E_{2}$ yields a logarithm in the leading term, but with a coefficient which does not depend on the sign of $\lambda$.

Gathering all the information obtained until now, we see that we can write $G$ in the form (for $r$ even)

$$
\begin{aligned}
G(\lambda)= & e^{-|\lambda|} \lambda^{\frac{r}{2}-1}\left\{\alpha \log |\lambda|+\theta(\lambda)\left[\sum_{0}^{N} c_{\varkappa}^{+} \lambda^{\varkappa / 2}+\log \lambda \sum_{1}^{N} d_{\varkappa}^{+} \lambda^{\varkappa / 2}\right]\right. \\
& \left.+\theta(-\lambda)\left[\sum_{0}^{N} c_{\varkappa}^{-}|\lambda|^{\alpha / 2}+\log |\lambda| \sum_{1}^{N} d_{\varkappa}^{-}|\lambda|^{\alpha / 2}\right]\right\} \\
& +O\left(\log |\lambda| \cdot|\lambda|^{\frac{r+N-1}{2}}\right)+s(\lambda)
\end{aligned}
$$

with $s \in \mathscr{S}$ and $N$ arbitrarily large. The coefficient $\alpha$ vanishes for definite $E_{2}$. The factor $e^{-|\lambda|}$ has been introduced to get a good behaviour at infinity. In the singular case the coefficients $\alpha$, etc., are functions $\in \mathscr{D}$ of $\boldsymbol{p}$, and thus integrable.

Analogous formulae hold for the derivatives of $G$. In particular, we have for the $O$-term in (58):

$$
\frac{\partial^{\beta}}{\partial \lambda^{\beta}} O\left(\log \lambda \cdot \lambda^{\beta_{0}}\right)=O\left(\log \lambda \cdot \lambda^{\beta_{0}-\beta}\right) \text { for } \beta<\beta_{0} .
$$


It is important to note that the $\psi_{\alpha}^{\mu}$, and hence the $\alpha, c_{\varkappa}^{ \pm}, d_{\varkappa}^{ \pm}$, vanish if $\psi$ vanishes in a neighborhood of $\mathscr{M}$. In this case $G$ is in $\mathscr{D}$.

We come now back to the asymptotic behaviour of $F(t)$ as defined by (32). The contribution of $s(\lambda)$ is strongly decreasing. For the $O$-terms we have, for $q<q_{0}=\frac{r+N-1}{2}$ :

$$
\left|t^{q} F_{O}(t)\right| \leqq \int d \lambda e^{-|\lambda|} O\left(|\lambda|^{q_{0}-q} \log |\lambda|\right)<\infty .
$$

The contributions of the remaining terms can be calculated explicitly, e.g.

$$
\begin{aligned}
\int_{0}^{\infty} d \lambda e^{i t \lambda} e^{-\lambda} \lambda^{\alpha} \log \lambda & \\
= & (1-i t)^{-1-\alpha}\left\{\int_{0}^{\infty} d \varrho e^{-\varrho} \varrho^{\alpha} \log \varrho-\log (1-i t) \int_{0}^{\infty} d \varrho e^{-\varrho} \varrho^{\alpha}\right\} \\
& \sim-(-i)^{-1-\alpha} \int_{0}^{\infty} d \varrho e^{-\varrho} \varrho^{\alpha} \cdot \log t t^{-1-\alpha}+\text { const. } t^{-1-\alpha}
\end{aligned}
$$

for large $t$.

The logarithms from the two $\alpha$-terms (for $\lambda \geqq 0$ and $\lambda \leqq 0$ respectively) cancel. Thus we obtain finally

$$
|F(t)| \leqq c|t|^{-r / 2} .
$$

For odd $k+l$ we are in the regular case $r=3(k+l-1)$ :

$$
|F(t)| \leqq c|t|^{-\frac{3}{2}(k+l-1)} .
$$

For even $k+l$ we may possibly be in the singular case, i.e. $r \geqq 3(k+l-2)$ :

$$
|F(t)| \leqq c|t|^{-\frac{3}{2}(k+l-2)} .
$$

If $\psi$ vanishes in a neighborhood of $\mathscr{M}$, then $F$ is strongly decreasing at infinity.

With (62) and (63) we can prove the estimate (21) at once. The HaagRuelle-condition follows then exactly as in the traditional proofs.

Let $\Phi(s)$ be defined by (18), with non-overlapping $\tilde{f}_{i}$. The vector $A_{f}(t) A_{f}^{*}(t) \Phi(s)$ is polynomially bounded in $s$ and $t$. This can be seen by expanding its squared norm in a cluster sum as was done in Eq. (22) and by noticing that $\tilde{W}^{T}$ is in supp $\tilde{f}$ a finite derivative of a continuous function [14]. Hence Hepp's proof [15] of the LSZ asymptotic condition is applicable to our case. 


\section{The Reduction Formulae}

In this section we shall make full use of condition $R$, not only of its corollary $P$.

Let $\chi\left(p_{2}, \ldots, p_{n}\right)$ be the distribution defined in condition $R$. For the sake of simplicity we consider the special case that $G_{\mu}$ is the ordinary retarded product $R$. Let $\tilde{f}_{j}(p) \in \mathscr{D}$, and define

$$
\begin{aligned}
\chi_{n}(p) & =\int \prod_{2}^{n-1}\left\{d p_{j} \tilde{f}_{j}\left(p_{j}\right)\right\} \chi\left(p_{2}, \ldots, p_{n}\right), \\
Y(t) & =\int d p \tilde{f}_{n}(p) \chi_{n}(p) e^{-i t p^{+}} .
\end{aligned}
$$

$\chi_{n}$ is regular in $\mathscr{T}_{-}$. According to Lemma 1 it can be written

$$
\chi_{n}(p)=\frac{\partial^{N}}{\partial p_{0}^{N}} \hat{\chi}(p)
$$

with $\hat{\chi}$ regular in $\mathscr{T}_{-}^{R}$ and continuous in $\operatorname{Im} p=0, N$ sufficiently large. Integration by parts yields

$$
Y(t)=\sum_{l=0}^{N} t^{l} \int d p \psi_{l}(p) \hat{\chi}(p) e^{-i t p^{+}}
$$

with $\psi_{l} \in \mathscr{D}$. Take any term of this sum. Let supp $\psi_{l} \subset\left\{\left|p_{0}\right|<x\right\}, 0<x<\infty$. The $p_{0}$-integration in (68) can then be extended over the compact intervall $-x \leqq p_{0} \leqq x$. Define

$$
\Psi\left(p_{0}, \boldsymbol{p}, t\right)=\frac{1}{2 \pi i} \int d q \frac{\psi_{l}(q, \boldsymbol{p})}{p_{0}-q} e^{-i t q} .
$$

$\Psi$ is as a function of $p_{0}$ (for real $\boldsymbol{p}$ and $t$ ) regular in the complex plane with the exception of a cut contained in $-x<p_{0}<x$.

For real $p_{0}$ define

so that

$$
\Psi^{ \pm}\left(p_{0}, \boldsymbol{p}, t\right)=\lim _{\varepsilon \rightarrow 0} \Psi\left(p_{0} \pm i \varepsilon, \boldsymbol{p}, t\right),
$$

$$
\psi_{l}(p) e^{-i t p_{0}}=\Psi^{-}(p, t)-\Psi^{+}(p, t) .
$$

We introduce an auxiliary function $\sigma(q) \in \mathscr{D}$, with $\sigma(q) \equiv 1$ in $|q| \leqq \varkappa$. The Fourier transform of $\sigma$ is called $\tilde{\sigma}$. We can write $\Psi^{+}$in the form

But

$$
\begin{aligned}
\Psi^{+}(p, t)= & \frac{1}{2 \pi i}\left\{\psi_{l}(p) \int d q \frac{e^{-i t q}}{p_{0}-q+i \varepsilon} \sigma(q)\right. \\
& \left.+\int d q \frac{e^{-i t q}}{p_{0}-q}\left[\psi_{l}(q, \boldsymbol{p})-\psi_{l}(p) \sigma(q)\right]\right\} .
\end{aligned}
$$

$$
\frac{1}{2 \pi i} \int d q \frac{e^{-i t q}}{p_{0}-q+i \varepsilon} \sigma(q)=\tilde{\sigma}(t) \theta(-t)
$$


i.e. the first term in (72) vanishes for $t>0$. For $\left|p_{0}\right| \leqq \varkappa$ the quotient in the second term is, considered as a function of $q$, a test function with compact support. The second term is the Fourier transform of this test function, hence strongly decreasing. Thus the $\Psi^{+}$-contribution to $Y$ decreases strongly for $t \rightarrow \infty$.

Both $\hat{\chi}(p)$ and $\Psi^{-}(p, t)$ are regular in $\operatorname{Im} p_{0}<0$. Therefore, we can deform the $p_{0}$-integration path in

$$
\int d^{3} p e^{-i t \omega(\boldsymbol{p})} \int_{-\varkappa}^{\varkappa} d p_{0} \hat{\chi}(p) \Psi^{-}(p, t)
$$

into a semicircle of radius $x$ in the lower half-plane. On this path we have $\Psi^{-}=\Psi$. The path is a compact set in the cut $p_{0}$-plane, and on such a set we have, for any positive integer $N$ :

$$
\begin{aligned}
t^{N}|\Psi(p, t)| & =\frac{1}{2 \pi}\left|\int d q \frac{\psi_{l}(q, \boldsymbol{p})}{p_{0}-q} \frac{d^{N}}{d q^{N}} e^{-i t q}\right| \\
& =\frac{1}{2 \pi}\left|\int d q e^{-i t q} \frac{d^{N}}{d q^{N}} \frac{\psi_{l}(q, \boldsymbol{p})}{p_{0}-q}\right| \\
& \leqq \frac{1}{2 \pi} \int d q\left|\frac{d^{N}}{d q^{N}} \frac{\psi_{l}(q, \boldsymbol{p})}{p_{0}-q}\right|<\infty
\end{aligned}
$$

uniformly in $p$. This means that $\Psi^{-}$decreases strongly on the deformed integration path, hence the $\Psi^{-}$-part of $Y$ decreases strongly for $t \rightarrow \infty$, and hence also $Y(t)$ itself.

As a side remark we note that the factor $e^{-i t \omega(p)}$ was of no consequence in these estimations. It could be replaced by a factor $e^{-i t(a, p)}$, $a$ arbitrary, without affecting the result. In this way we see that the retarded function $r^{\prime}\left(x_{1}-x_{2}, \ldots, x_{1}-x_{n}\right)$ decreases strongly in the directions $\left(x_{1}-x_{j}\right) \notin \bar{V}_{+}$for a $j \geqq 2$. This property replaces the support condition of the localizable case.

From the above estimate and some straightforward generalizations we obtain with the help of the algebraic relations between the retarded products among themselves and with the $T$-products [6] the following facts $(\tilde{f} \in \mathscr{D})$ :

$$
\begin{gathered}
\lim _{t \rightarrow \infty} \int d p_{n} \tilde{f}^{*}\left(p_{n}\right) \tilde{R}\left(p_{1}, \ldots, p_{n}\right) e^{-i t p^{-}}=0, \\
\lim _{t \rightarrow \infty}\left\{\int d p_{n} \tilde{f}^{*}\left(p_{n}\right) R\left(p_{1}, \ldots, p_{n}\right) e^{-i t p^{-}}-\left[R\left(p_{1}, \ldots, p_{n-1}\right), A_{f}(t)\right]\right\}=0 \\
\lim _{t \rightarrow-\infty}\left\{\int \prod_{1}^{n}\left[d p_{j} \tilde{f}_{j}^{*}\left(p_{j}\right) e^{-i t p_{\bar{j}}}\right] \tilde{T}\left(q_{1}, \ldots, q_{m}, p_{1}, \ldots, p_{n}\right)\right. \\
\left.-\tilde{T}\left(q_{1}, \ldots, q_{m}\right) \int \prod\left[d p_{j} f_{j}^{*}\left(p_{j}\right) e^{-i t p_{\bar{j}}}\right] \tilde{T}\left(p_{1}, \ldots, p_{n}\right)\right\}=0 .
\end{gathered}
$$


With the help of these relations we can easily transcribe the proofs of the LSZ reduction formulae based on the Haag-Ruelle asymptotic condition [6] or the LSZ asymptotic condition [15] for our case. The proof [15] that the amputated $\tilde{\tau}^{T}$ are, for non-overlapping momenta, continuous in the variables $p_{j}^{+}$in a neighborhood of the mass shell, also carries over to our case with some obvious modifications.

The result is, that the LSZ reduction formulae hold in exactly the same form as in localizable theories.

\section{References}

1. Streater, R. F., Wightman, A. S.: PCT, spin + statistics, and all that. New York: W. A. Benjamin Inc. 1964.

2. Jost, R.: The general theory of quantized fields. Providence: Am. Math. Soc. 1965.

3. Jaffe, A.: Phys. Rev. 158, 1454 (1957).

4. Güttinger, W.: Nuovo Cimento 10, 1 (1958).

5. Informal meeting on renormalization theory. Report no. IC/69/121, International Centre for Theoretical Physics, Trieste 1969.

6. Steinmann, O.: Commun. Math. Phys 10, 245 (1968).

7. Gel'fand, I. M., Shilov, G. E.: Generalized functions, vol. 1, chapter 2. New York: Academic Press 1964.

8. Epstein, H., in: Axiomatic field theory, ed. M. Chrétien and S. Deser, New York: Gordon and Breach 1966.

9. Steinmann, O.: Helv. Phys. Acta 33, 347 (1960).

10. Ruelle, D.: Nuovo Cimento 19, 356 (1961).

11. Haag, R.: Phys. Rev. 112, 669 (1958).

12. Ruelle, D.: Helv. Phys. Acta 35, 147 (1962).

13. Hepp, K.: Helv. Phys. Acta 37, 639 (1964).

14. Schwartz, L.: Théorie des distributions, p. 82. Paris: Hermann 1966.

15. Hepp, K.: Commun. Math. Phys. 1, 95 (1965).

O. Steinmann

Schweizerisches Institut f. Nuklearforschung

CH-8044 Zürich, Hochstr. 60 